\title{
El impacto educativo de los programas intergeneracionales: un estudio desde la escuela y las diferentes instituciones sociales implicadas
}

\author{
The educational impact of the intergenerational programmes: \\ a study from the school and different social institutions involved
}

\author{
Pedro Moreno Abellán; Silvia Martínez de Miguel López;Andrés Escarbajal de Haro \\ Universidad de Murcia, España
}

\section{Resumen}

El presente artículo analiza el impacto de los programas intergeneracionales desde la escuela y las diferentes instituciones sociales para la mejora y calidad de futuros proyectos que generen la construcción de nuevos aprendizajes, y fortalezca un acercamiento entre generaciones. Para abordarlo, se han descrito y analizado los programas orientados al ámbito de intervención intergeneracional en sus diferentes contextos de actuación, las funciones y características de todos los agentes implicados en la acción intergeneracional, y se han identificado las carencias desde la percepción que tenían los protagonistas de esos programas. Para atender a los aspectos planteados, se ha llevado a cabo una metodología de carácter cualitativo a través de entrevistas semiestructuradas y grupos de discusión. Entre los principales resultados obtenidos se reconoce la satisfacción personal de los mayores por el hecho de sentirse útiles y seguir aprendiendo a través de estas iniciativas. En el caso de los niños entrevistados que interactúan con mayores, se configuran beneficios relacionados esencialmente con el conocimiento y la sabiduría que les aportan esas personas; adquisición de numerosos valores de empatía, respeto y solidaridad; aprender a ser mejores personas en la vida; disminuir estereotipos hacia la vejez; y reconocer una imagen positiva de las personas mayores.

Palabras clave: niños; personas mayores; programas intergeneracionales; envejecimiento activo; participación social.

\begin{abstract}
The present article analyses the impact of the intergenerational programmes from the school and different social institution to improve and offer more quality for design of futures projects which generate the construction and exchange of new learning. More specifically to improve the strength between the connection of every generation. With this aim in mind, has been described and analysed the existence of programmes about intergenerational fields in the different contexts, the functions and characteristics regarding all the participants involved, and the existence of certain lacks on the part of the protagonists involved in intergenerational programmes. To attend to the purposes previously shown, a qualitative methodology was used through semi-structured interviews and group discussions. Finally, among the main results discovered, between other aspects, personal satisfaction is the biggest demonstrated by the fact of feeling useful and of keeping on learning. In case of the interviewed children who interact with elderly people, there are formed benefits related essentially to the knowledge that they bring them; acquisition of numerous values of empathy, respect and solidarity; to learn to be better people in the life; to diminish stereotypes towards old age; and to recognize a positive image of the elderly.
\end{abstract}

Keywords: elderly; intergenerational programmes; active ageing; social participation. 


\section{INTRODUCCIÓN}

La sociedad actual se encuentra sometida a numerosos factores de cambio; uno de los mayores es, sin duda, la profunda transformación demográfica que se otea en el horizonte como un enorme desafío para el siglo XXI. En ese sentido, el 'envejecimiento demográfico' quiere ser 'tratado' desde la teoría y la práctica del envejecimiento activo, optimizando las oportunidades de bienestar físico, social y mental de las personas mayores con el objetivo de ampliar la calidad de vida en la vejez. De este modo, calidad de vida y envejecimiento activo estarían tan imbricados que trascienden el plano teórico para adentrarse en objetivos y desarrollo prácticos, y apunta claramente hacia la autonomía y la calidad de vida en su doble vertiente: responsabilidad de acción y compromiso de participación en diferentes espacios sociales (Del Valle y Coll, 2011). Pero, para conseguirlo, es necesario vehicular nuevos instrumentos y estrategias para intervenir socioeducativamente con el colectivo de personas mayores. De igual forma, se hace necesario fomentar y asentar la equidad en la participación social del colectivo de personas mayores respecto al resto de grupos de edad.

Al mismo tiempo, es imprescindible una nueva concepción de espacios propios de participación en el que confluyan diferentes cohortes de la esfera social, espacios interactivos e intergeneracionales (a partir de las personas mayores como principal cohorte), que es una demanda ineludible que todas las instituciones, ya sean administrativas o técnicas, deben apoyar, regular normativamente y ampliar para ofrecer nuevos modelos de motivación por y para este colectivo. Para la propia Organización Mundial de la Salud (OMS,2002), la solidaridad intergeneracional significa "dar y recibir de manera recíproca entre individuos, así como entre generaciones de mayores y jóvenes" (p. 79). Ello comporta la equidad entre las generaciones, pero también el compromiso de construir juntos el futuro; es un elemento de solidaridad clave en todos los tiempos y en todas las sociedades, pero mucho más desde planteamientos modernos de envejecimiento activo (Baschiera, Deluigi \& Luppi, 2014). Y desde el punto de vista educativo, con esas medidas se haría realidad el inseparable proceso de enseñanza-aprendizaje a lo largo de todo el proceso vital.

De acuerdo con lo anterior, cobra todo el sentido la alternativa de las relaciones intergeneracionales, y en consecuencia, a los programas de carácter intergeneracional, como vía creciente de participación para las personas mayores y las generaciones más jóvenes dentro del conjunto social. Una fuente de estudio 
que suscita gran interés en la actualidad para la mejora y calidad de vida, la autoestima y la dignidad de los mayores; donde especialmente este colectivo signifique un pilar fundamental para las demás generaciones. En este sentido, los Programas Intergeneracionales (en adelante PI) pueden ser un instrumento de gran viabilidad, tanto en la participación de personas mayores para que sus voces puedan ser escuchadas, como en los beneficios que conllevan para las generaciones más jóvenes, que a su vez, pueden recibir experiencias, construir conocimientos y revalorizar la imagen del colectivo de mayores.

Los primeros PI que surgen en Estados Unidos durante la década de los años sesenta pretenden concienciar y paliar el distanciamiento familiar de los jóvenes, generado por los cambios producidos en el contexto laboral (Newman y Sánchez, 2007). Hasta los años noventa en el contexto americano, se produce un evolución en la que se desarrollan principalmente problemáticas culturales y sociales. Es a partir de esta década, cuando los PI se expanden al continente europeo para ser utilizados como instrumento de avance educativo y social. Según mantienen los autores, ofrecen una respuesta a la inclusión de las personas mayores ante el envejecimiento activo, y a los nuevos modelos de solidaridad familiar y las políticas de integración centradas en los procesos migratorios.

La primera conceptualización que fortalece la evolución de los PI, viene marcada por el International Consortium of Intergenerational Programmes al definirlos como "vehículos para el intercambio determinado y continuado de recursos y aprendizaje entre las generaciones mayores y las más jóvenes con el fin de conseguir beneficios individuales y sociales" (ICIP, 1999; Hatton-Yeo y Ohsako, 2001). Reflejo de ello, es la implicación de distintas generaciones en actividades comunitarias para la consecución de unos beneficios recíprocos. En este sentido, diversas investigaciones (Gutiérrez, 2010; Mcrea y Smith 1997), demuestran un impacto positivo ante la importancia de cubrir necesidades sociales y crear numerosos lazos afectivos entre niños y mayores.

En el caso de niños y jóvenes, recae sobre el carácter educativo a través de programas de mentorización, de tutorización o para evitar el absentismo escolar. Pinazo (2009) y MacCallum, et al. (2006), subrayan la responsabilidad social, la confianza en uno mismo o el hecho de retirar ciertos estereotipos negativos hacia la vejez. Para las personas mayores (Gutiérrez, 2010), es una oportunidad para seguir aprendiendo y ofrecer su experiencia vivida convirtiéndolas en personas 
activas. De la misma manera, el estudio de Gutiérrez y Hernández (2013) demuestra el bienestar psicológico, físico y social. Respecto al impacto de los participantes mayores en $\mathrm{Pl}$, es concluyente que el contacto con niños y jóvenes provoca un sentimiento de vitalidad, un aumento de autoestima, así como la reducción del aislamiento y la soledad, para dar paso a un envejecimiento saludable.

En cuanto a la esfera comunitaria, aparecen grandes beneficios relativos a la creación de redes sociales para la integración multi-generacional (Granville y Hatton-Yeo, 2002; Sánchez, Díaz, López y Sáez, 2008; Gutiérrez y Hernández, 2013). También se incide en la consolidación de sociedades del futuro entendidas como un espacio intergeneracional de participación activa y educación social (Martínez de Miguel, Escarbajal y Moreno, 2017). Dentro de la literatura intergeneracional, Kuehne (2003) remarca la importancia de implementar otros estudios de mayor envergadura, que incluyan otras generaciones dentro del ámbito familiar, con objeto de comprobar una nueva repercusión sociocomunitaria. De forma análoga, Hatton-Yeo (2009) ofrece valor a la creación de PI de carácter comunitario, centrados más bien, en políticas que garanticen la equidad social. En consonancia, el estudio de Villas-Boas, Oliveira, Ramos y Montero (2015), distingue la importancia del impacto comunitario como otro elemento de estudio, además de la implicación de las principales generaciones implicadas.

Como apuntan Salmerón, Martínez de Miguel y Escarbajal (2014), es fundamental que "la información sobre las personas mayores también llegue a los jóvenes, y que llegue libre de sesgos sociales, de imposiciones, de ideas preconcebidas..." (p. 152), para entender que el proceso de envejecimiento no es un compartimento estanco. Estas investigaciones, se desarrollan precisamente desde el convencimiento de que, a través de los PI puede garantizarse el diálogo entre generaciones, donde se traten determinados temas influyentes sobre la inclusión social; marcando un escenario progresivo de participación activa y de extensión de redes sociales de todos y para todos. Por ello, consideramos que a través del diseño y puesta en marcha de PI se puede contribuir de manera positiva al desarrollo de la educación para la ciudadanía en su sentido más amplio. Demostrando así, que estos programas contribuyen a la promoción de solidaridad social y a la proyección ciudadana entre generaciones (Pinazo, 2012). 


\subsection{Objetivos}

Para la presente investigación se plantearon los objetivos que siguen a continuación:

\section{Objetivo general}

- Conocer el estado de los PI en la Región de Murcia desde el punto de vista de sus participantes

\section{Objetivos específicos}

- Analizar el impacto de los programas orientados al ámbito de intervención intergeneracional en sus diferentes contextos de actuación (Centros Sociales, Colegios, Ayuntamientos...), así como los motivos de la participación en los mismos.

- Conocer las funciones y características de los agentes implicados en la acción intergeneracional: niños, personas mayores y técnicos.

- Identificar la existencia de determinadas carencias en los programas intergeneracionales.

\section{METODOLOGÍA}

En esta investigación se ha optado por la utilización de una metodología cualitativa, pues se defiende la idea de que la mejor forma de llegar a tener un mayor conocimiento del mundo que rodea a las personas mayores es a través de las voces de los propios protagonistas: las personas mayores y otros agentes sociales relacionados con ellos (Serdio, Díaz y Cifuentes, 2013).

\subsection{Población y muestra}

La población estudiada se encuentra distribuida de manera equilibrada por toda la geografía de la Región de Murcia, siendo los escenarios principales, y, por tanto, el criterio de selección, aquellos Centros Sociales que desarrollaran un mayor número de PI (Murcia capital, Alcantarilla, San Javier y Puerto de Mazarrón), así como en centros educativos (con niños pertenecientes a $5^{\circ}$ y $6^{\circ}$ curso de Educación Primaria). Este contexto se amplía a profesionales y/o coordinadores de PI, procedentes de otros municipios como Lorca, Archena, Molina de Segura, Cartagena, Caravaca, Jumilla, La Unión, Las Torres de Cotillas y Yecla. En la Tabla 1, se muestra resumidamente los principales aspectos sociopersonales en relación al número de participantes, sexo, años de participación y la totalidad de participantes del estudio. 
TABLA 1

Principales aspectos sociopersonales del conjunto total de participantes

\begin{tabular}{lcccccc}
\hline \multicolumn{2}{c}{ Colectivos de estudio } & \multicolumn{2}{c}{ Sexo } & \multicolumn{3}{c}{ Años de participación } \\
\hline \multicolumn{1}{c}{ Grupo } & $\begin{array}{c}N^{0} \\
\text { participantes }\end{array}$ & m. & fem. & $\begin{array}{c}\text { Hasta } \\
5 \text { años }\end{array}$ & $\begin{array}{c}\text { Entre } \\
6-10 \text { años }\end{array}$ & $\begin{array}{c}\text { Más } \\
\text { de } 10 \text { años }\end{array}$ \\
\hline Personas mayores & 28 & 14 & 14 & 8 & 12 & 8 \\
Profesionales & 18 & 4 & 14 & 1 & 6 & 11 \\
Niños & 50 & 22 & 28 & 50 & - & - \\
Total participantes & 96 & 40 & 56 & 59 & 18 & 19 \\
\hline
\end{tabular}

Fuente: elaboración propia.

\subsection{Instrumentos}

Los instrumentos de recogida de información utilizados en esta investigación han sido la entrevista y el grupo de discusión. En el caso de la entrevista, se optó por una semiestructurada, elaborando dos modelos: por un lado, la dirigida a profesionales coordinadores, fundamentada en el modelo de entrevista para profesionales elaborada por Martínez de Miguel (2003); por otro lado, para alumnos de Educación Primaria implicados en PI con personas mayores, ésta muy clara y sencilla, adaptada a su edad, teniendo como referencia y ajustando hacia un enfoque cualitativo el "Cuestionario sobre las Relaciones Abuelos-Nietos" de Rico, Serra, Viguer y Meléndez (2000). En los grupos de discusión participaron las personas mayores, que podían matizar, ampliar, comparar percepciones, informaciones, sentimientos, emociones, etc., resultando ser una estrategia investigadora de recogida de información muy significativa.

\subsection{Procedimiento de recogida y análisis de datos}

Tanto en el proceso de realización de las entrevistas individuales como grupales (para grupo de discusión), se consideró adecuado que el investigador se desplazara a los diferentes centros. Asimismo, se garantizó en la primera toma de contacto que la información recabada sería utilizada exclusivamente para dichos fines de investigación. En cuanto a las sesiones individuales para las entrevistas a los niños, se optó por realizarlas en el aula correspondiente de cada centro educativo, a excepción, de un pequeño grupo de niños que realizó la entrevista en uno de los Centros sociales, aprovechando dos días de convivencia programados con las personas mayores, dentro del PI desarrollado en ese momento. Para las sesiones grupales se propuso como espacio más adecuado la sala de juntas de los diferentes Centros Sociales; este proceso de recogida de información fue grabado en el mismo momento de la realización del grupo de discusión con un tiempo estimado de hora y media. 
Para el proceso de análisis de datos, y tras la grabación de entrevistas y grupos de discusión, se procedió a la transcripción de cada uno de los relatos haciendo uso de una lectura reflexiva. Seguidamente se establecieron una serie de códigos para encuadrar todos los apartados de forma ordenada en los que se pretendía focalizar el análisis. A partir de aquí, se procedió a la construcción de categorías descriptivas que permitieran detallar y estructurar los núcleos temáticos o unidades de codificación sin olvidar el sentido global. Posteriormente, se optó por uno de los procedimientos habituales en investigación cualitativa a la hora de exponer los resultados como es el uso del texto narrativo para realizar un análisis de contenido. La finalidad radicaba en tratar de organizar y dar significatividad a las categorías de información establecidas.

\section{RESULTADOS}

Teniendo en cuenta los diferentes núcleos temáticos, se establecieron finalmente cuatro categorías: motivos de participación, contenido de los PI, rol desempeñado y necesidades percibidas en los PI desde la perspectiva de sus protagonistas. A continuación, en las Figuras 1 y 2, se exponen los resultados, haciendo uso posteriormente del texto narrativo.

\section{Motivos de participación}

FIGURA 1

Motivos de participación de las personas mayores en PI

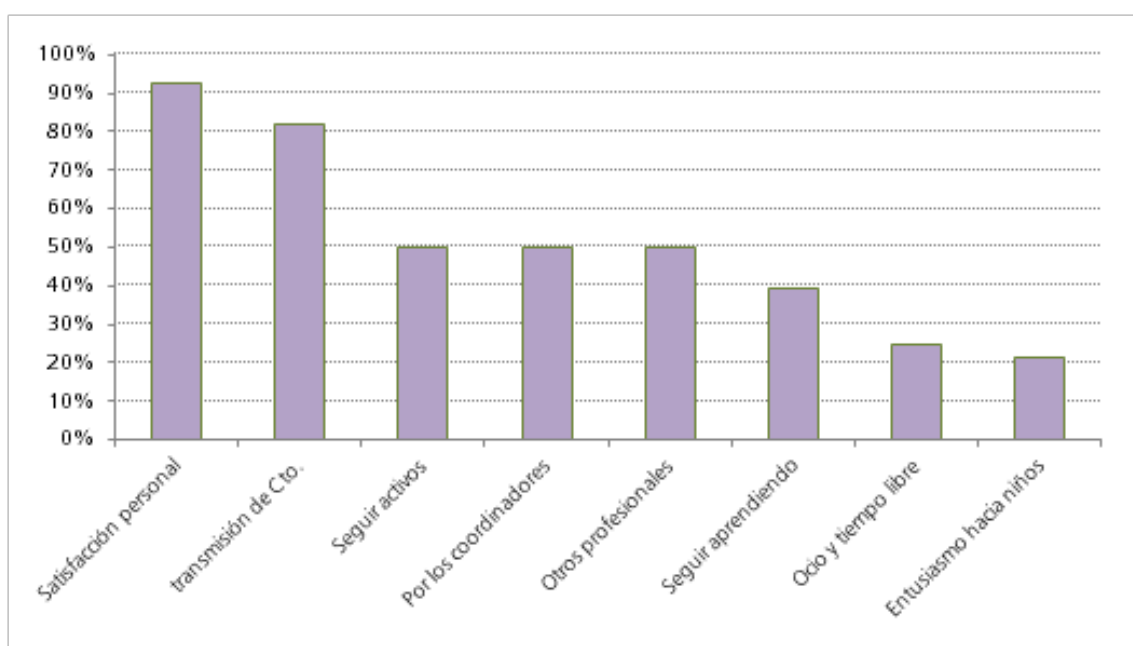


FIGURA 2

\section{Motivos de participación de los coordinadores en PI}

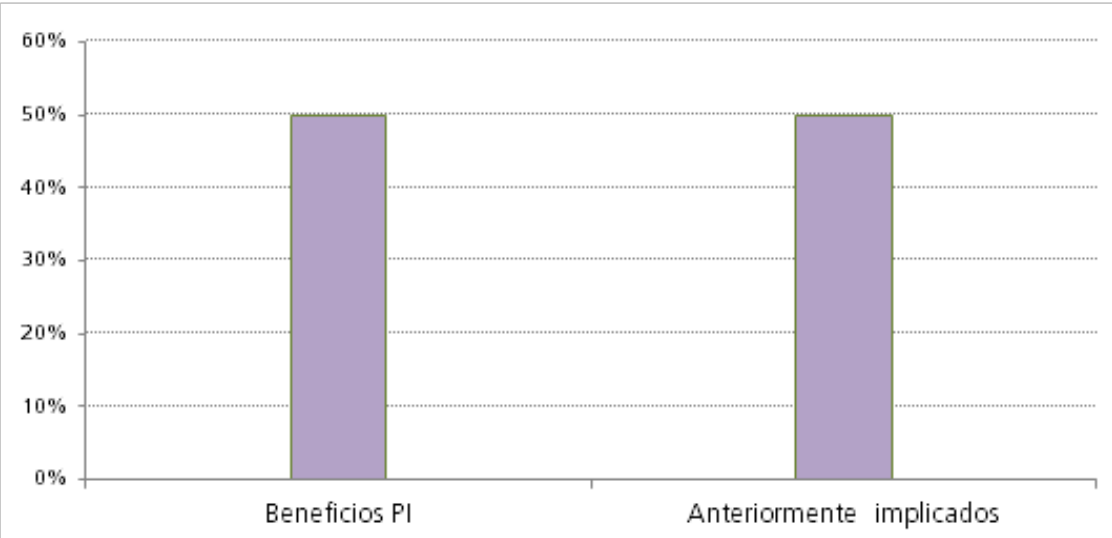

Con esta categoría se pretende descubrir los motivos e intereses que han impulsado a los protagonistas para participar en los Pl. Así, se constata, que para la gran mayoría de las personas mayores, los principales motivos de participación corresponden a su satisfacción e iniciativa personal: Fue la idea nuestra. Y ya luego al ayuntamiento le pareció bien y entonces nos mandó una asistenta que estaba metida (PMGD1A). Seguida de esta gran mayoría, otra razón de participación la constituye el hecho de que los mayores expresan la importancia de que niños y jóvenes, de alguna manera, conozcan su mundo al tiempo de transmitirle su conocimiento: Que los muchachos puedan entender un poco nuestro mundo porque hay un abismo entre la mentalidad que ellos tienen y nosotros con nuestros setenta años (PMGD1A). Por otra parte, la mitad de los mayores participantes opinan que el hecho de participar en este tipo de programas les hace seguir activos. Una manera de sentirse útiles consigo mismos, renovarse, y poder compartir su experiencia con sus iguales u otras generaciones: Mayor parte por el envejecimiento activo.(PMGD4A).

En la misma medida, se descubren nuevas formas de involucrar a los mayores. A través de los coordinadores del propio PI: La verdad que el programa que llevamos este año que lo ha preparado Montse. Que ella es la culpable (PMGD1A). Por la motivación de otros mayores que ya participaban con anterioridad: Porque yo fui a aprender internet y me vistieron... (PMGD8J). Por la recomendación de otro profesional del centro no implicado: Porque dice la psicóloga que asistas a clase, porque deberías quedarte a ayudarnos. Total, que ya me van enrollando (PMGD9J). Y, en menor medida, para seguir aprendiendo: Estamos en edad todavía de aprender (PMGD5A). Hubo un pequeño grupo 
que manifestó su iniciativa de participación, como la mejor manera de utilizar su ocio y tiempo libre tras la jubilación: Y he descubierto después de jubilado, que existe el tiempo (GDPMU27). También se identifica que durante su etapa de trabajadores no tenían la misma disponibilidad que ahora: Antes no tenía tiempo de nada trabajando (GDPMU27). Otro grupo de participantes enfoca su motivo de participación por medio del entusiasmo y cariño que sienten hacia los niños: A mí, te digo claramente, es porque me encantan los niños (PMGD8J).

En el caso de los profesionales coordinadores del programa, los motivos de participación hacen referencia, principalmente, a los beneficios encontrados a través de los PI: Cuando realizábamos semanas culturales en centros residenciales y vimos lo positivo que resultaba el contacto de niños en esos ámbitos cerrados para los mayores (EPF12). Otra de las razones de participación se debe a que los programas ya estaban implantados: Cuando comencé a trabajar en el centro de mayores, éste era uno de los programas que ya estaba instaurado (EPF8). Finalmente, en relación a los motivos de participación de los niños, a la inmensa mayoría les motiva el hecho de adquirir valores y nuevos aprendizajes: La verdad que cuando estoy con ellos aprendo muchas cosas diferentes (N5); A convivir con los mayores (N22); también, porque se divierten con los mayores: Que son muy divertidas (N16).

\subsection{Contenido de los programas intergeneracionales}

A partir de esta categoría se trata de descubrir qué actividades se suelen ofrecer dentro de estos programas, a qué grupos suelen dirigirse, cuáles se dan con mayor frecuencia, espacios y tiempos, por qué esas actividades y no otras atendiendo a su criterio de selección, desarrollo, objetivos y evaluación del proceso, y cómo nacen o de qué manera se fundamentan dentro del proceso de organización (Véase tabla 2).

\section{TABLA 2}

\section{Grupos de edad y contextos de interacción}

\begin{tabular}{ll}
\hline \multicolumn{1}{c}{ Grupo de edad } & \multicolumn{1}{c}{ Contexto de interacción } \\
\hline Educación Infantil & Guarderías (hasta 3 años) \\
Educación Primaria & Centros escolares \\
Educación Secundaria & Institutos \\
Educación Universitaria & Centros universitarios \\
Otros grupos de Mayores & Centros Sociales \\
Trabajador social procedente de otro centro de mayores & Centros Sociales \\
\hline
\end{tabular}


De la anterior tabla se desprenden los grupos de participación con los que interactúan las personas mayores. Así, tal y como remarcan mayores y coordinadores, el principal grupo de edad en el que poseen una dilatada experiencia de trabajo, hace referencia a los niños de Educación Primaria en Centros escolares: Llevamos más menos, unos 7 u 8 años... fuimos a los colegios, pero fuimos a los colegios a darles charlas de nuestras experiencias (GDPMU24). Aunque, en menor medida, se han programado actividades con alumnado de educación secundaria en diferentes institutos: Hemos ido escalando, primero a los colegios, luego hemos ido a los institutos (GDPMU27). Igualmente, se han llevado a la práctica actividades en guarderías: Yo lo que quería decir es que también participábamos en guarderías con bebes, con niños de dos tres años (PMGD12J). Seguidamente, se encuentra otro tipo de programa que hace referencia a las jornadas intergeneracionales con alumnos universitarios de educación social: Otro, asistencia a la universidad como hemos dicho antes (PMGD4A). Asimismo, los profesionales resaltan su coordinación e implicación: En la actualidad llevo el seguimiento del Taller de la Experiencia y de la Universidad de Educación social que nos hizo partícipes en este gran proyecto (EPF7). Por último, aunque en menor medida, los profesionales han desarrollado experiencias con otros colectivos como discapacitados, familia, o acciones comunitarias.

Prestando atención a la relación mayores-niños, se observa que el centro escolar es el contexto habitual de actuación para la mayoría de las actividades programadas. El eje vertebral está integrado por el Taller de la experiencia, que se enmarca dentro de las siete áreas que conforman el denominado "ámbito socio-comunitario": En las experiencias intergeneracionales dando las charlas (PMGD6A). Aquí los mayores cuentan su experiencia desarrollando múltiples actividades de forma interactiva con el alumnado, reservando un espacio de pregunta-respuesta: Es decir, luego se hace una especie de coloquio en el que los niños pueden preguntar (GDPMU23).

Dentro del conjunto de actividades, y tras el análisis de los grupos de discusión de personas mayores, se contempla con gran éxito los juegos tradicionales y la fabricación de juguetes: Por ejemplo, a jugar a los bolos, al caliche, a una demostración de esparto (GDPMU26). En el caso de los niños, la actividad más valorada es el cuentacuentos: Que nos cuenten cuentos (N7); seguida de actividades de juegos antiguos y tradicionales: La petanca y los bolos (N3). No menos importante es el proceso de evaluación para comprobar el nivel de satisfacción de las actividades: Y luego, las hojas que le dan alli los maestros, 
se las mandan de aqui preparadas, ellos las rellenan con las preguntas que le den y unos dicen unas cosas que les gustan más..., otros dicen otras, pero que lo meten todo (PMGD15Z).

En cuanto a la planificación, los mayores preparan y dan forma a los temas previa exposición: El tenía cuatro temas, yo otros cuatro temas, y cada uno se va haciendo sus temas que va desarrollando en el colegio donde vamos (GDPMU27). Del mismo modo, se reúnen previamente con los coordinadores del programa para la elección de los temas: Se elegían los temas (GDPMU22); Los llevamos dentro, son los temas que nosotros creemos (GDPMU27), como también, comentar y matizar entre todos aquellos aspectos susceptibles de mejorar y poder concluir en el tema que finalmente se trasladará a la práctica: Los exponemos en las reuniones que tenemos aquí cada semana, y los compañeros, pues nos dan o damos el visto bueno al trabajo que hacemos cada uno (GDPMU28).

\subsection{Rol desempeñado}

Esta categoría pretende revelar la forma de implicación, el tipo de funciones llevadas a cabo durante el desarrollo del programa, así como prever todas aquellas actuaciones significativas antes y después del mismo en las que pueden involucrarse activamente. Se constata que la gran mayoría de personas mayores cumplen una importante función de transmisión útil para los niños: Lo que yo intentaba era llevar a la mente de los chavales el sentido de la responsabilidad (PMGD4A). Las personas mayores consideran que cumplen un papel activo; se sienten satisfechas y protagonistas en el desarrollo de las actividades: Nuestro rol yo sí que lo veo activo. No paramos ni un segundo (PMGD19Z). A estas afirmaciones, se une también la percepción positiva de los mayores desde el punto de vista de los niños: Pues que los mayores pueden hacer cosas que ni te imaginas (N21).

De otro lado, y dentro de ese papel activo casi generalizado, se constata que más de la mitad de los participantes se consideran protagonistas de la organización y planificación del programa llevado a cabo: Nosotros empezamos solitos, eh. $Y$ sin nadie (PMGD1A). Asimismo, aunque la idea nace de ellos, se afirma, que más tarde recibieron la participación de otros agentes: Fue la idea nuestra. Y ya luego al ayuntamiento le pareció bien y entonces nos mandó una asistenta que estaba metida (PMGD1A). Preparan y eligen previamente los temas que van a exponer y los elaboran ellos mismos; llegando a matizarlos de forma conjunta: Elaboramos los temas que vamos a dar y los exponemos, lo único es que aquí los purificamos... (GDPMU27). No obstante, los profesionales aportan la 
supervisión técnica, independientemente de que los vean capaces de desarrollar las actividades por sí mismos: Las personas mayores son capaces... bajo supervisión técnica y dentro de un proyecto con objetivos definidos (EPF5). Véase a continuación la Figura 3, que resume el rol desarrollado por los mayores:

\section{FIGURA 3}

\section{Rol desempeñado por las personas mayores en PI}

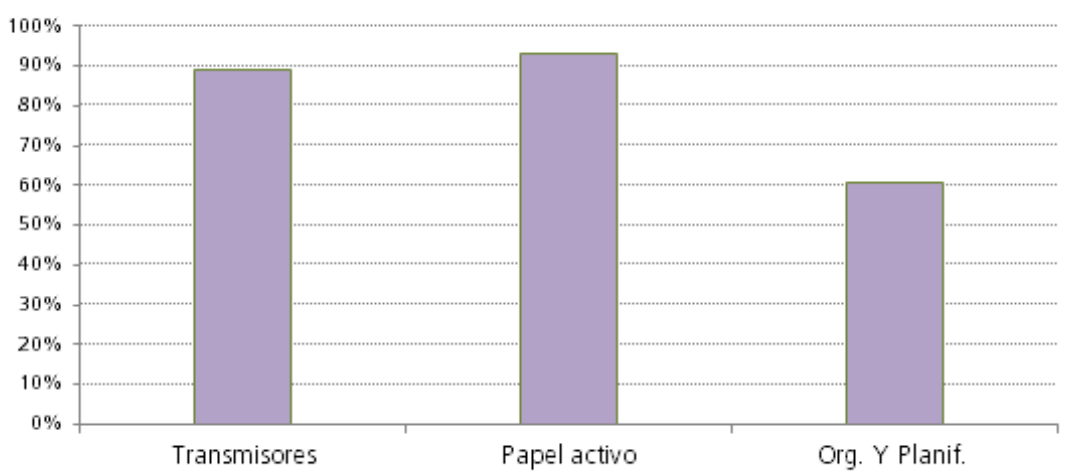

$42 \quad$ Necesidades percibidas en los PI

Esta última categoría que analiza las necesidades presentes en el desarrollo de los $\mathrm{Pl}$, está conformada por dos subcategorías: la primera versa sobre los aspectos formativos; la segunda, engloba carencias de tipo estructural, administrativo y económico. Obsérvese previamente en las Figuras 4 y 5 :

\section{FIGURA 4}

\section{Necesidades percibidas por las personas mayores en PI}

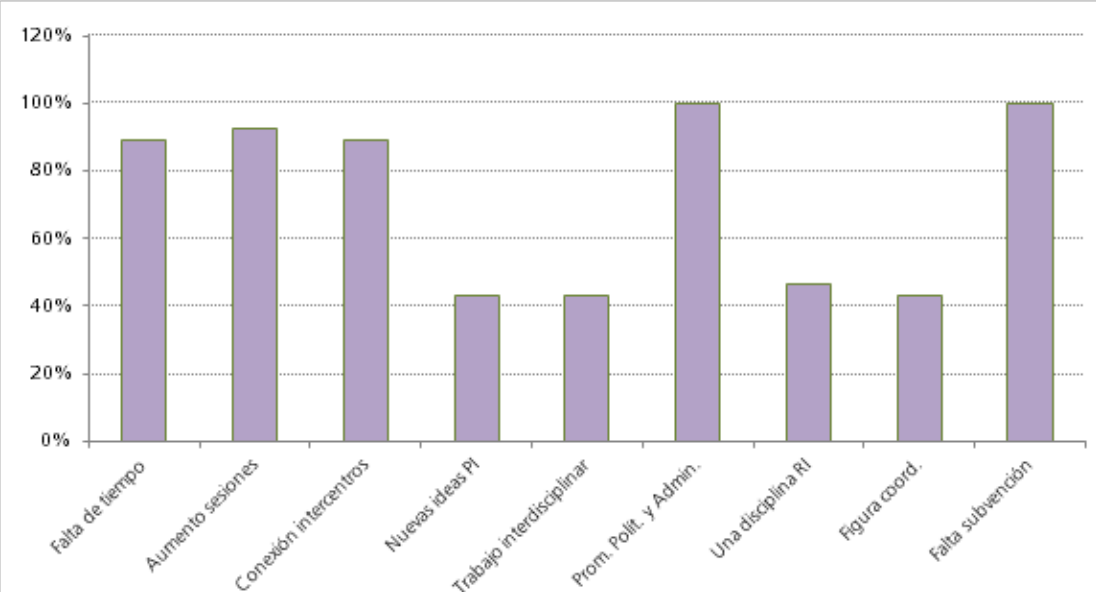




\section{FIGURA 5}

\section{Necesidades percibidas por los coordinadores en PI}

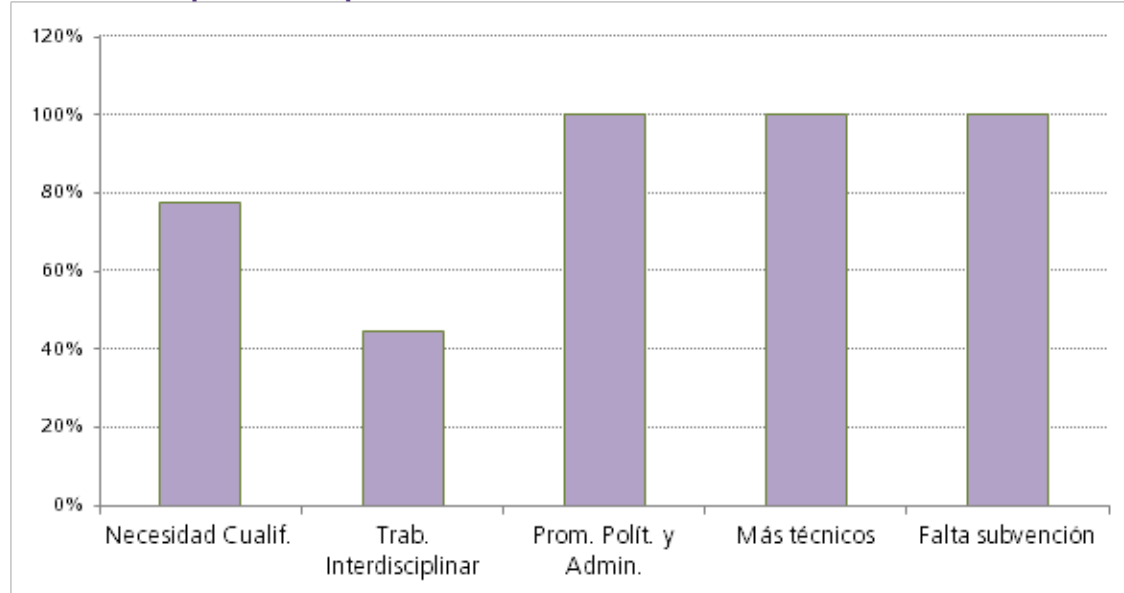

a) Necesidades formativas

Un grupo significativo de participantes mayores expresa su falta de aproximación a las nuevas tecnologías como apoyo al desarrollo del programa, pero también para adaptarse a los 'nuevos tiempos': Yo creo, que como el sistema educativo ha cambiado tantísimo, con las nuevas tecnologías..., y además de que esa parte de pizarras electrónicas (PMGD3A). Desde la perspectiva de los profesionales, y a pesar de que muchos se han ido formando con el transcurso de los años, siguen manifestando la necesidad de cualificación en este ámbito: Falta formación para aquellas personas que realmente se quieran dedicar a las relaciones intergeneracionales (EPF1).

De otro lado, los mayores descubren en los niños algunos prejuicios hacia las personas de edad avanzada, aunque posteriormente van desapareciendo una vez desarrollado el programa: Porque al principio piensan que no vamos a saber hacer muchas cosas, y luego se sorprenden cuando nos ven ahi de pie dando nuestra charla (GDPMU22). Coincidiendo con los mayores, casi la mitad de los niños entrevistados percibía una imagen negativa de aquéllos antes de interaccionar con ellos; y solamente un grupo reducido poseía una imagen positiva, con alguna connotación negativa. Véase la Figura 6: 


\section{Imagen previa a la interacción}

FIGURA 6

Imagen de las personas mayores por niños antes de la interacción en PI

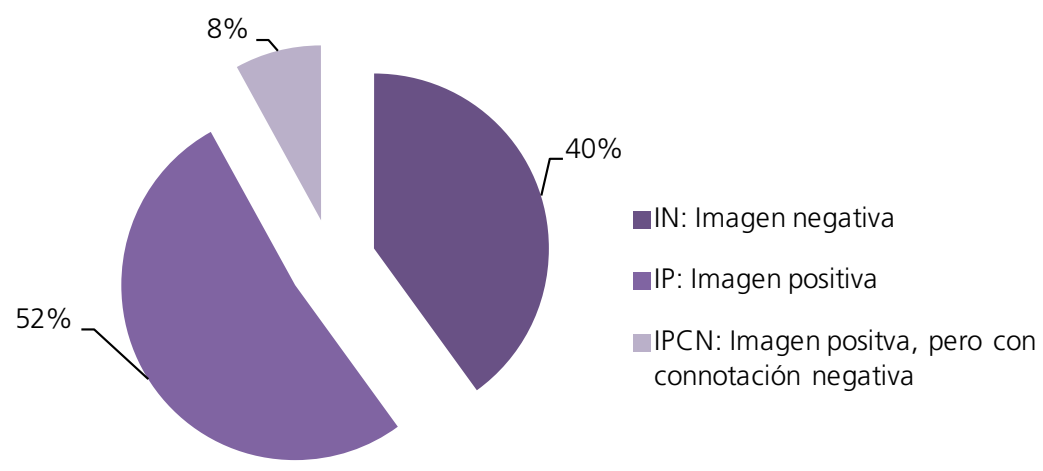

Comparando los anteriores resultados con la imagen que los niños poseen de los mayores después de la interacción, se descubre entre otros aspectos, que esa visión negativa cambia a positiva de manera considerable tal y como se muestra en la Figura 7:

\section{Imagen posterior a la interacción}

FIGURA 7

Imagen de las personas mayores por niños después de la interacción en PI

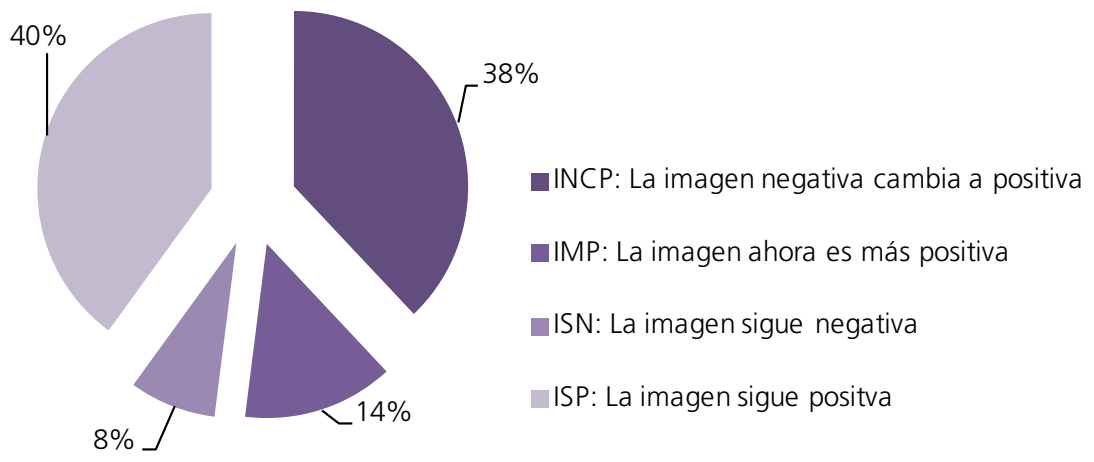


Finalmente, cabe expresar el desconocimiento del proceso de ejecución de los PI por parte del profesorado: El profesor está allí. Para ellos es una cosa que desconocían. Porque también son personas jóvenes, desconocen esa actividad y piensan, -ah, pues qué bien, mira estos lo que hacen, que estupendo (PMGD3A). Esta expresión refleja la necesidad de ofrecer mayor información e implicación al profesorado de los centros escolares.

b) Necesidades estructurales, administrativas y económicas

Atendiendo a las necesidades estructurales, se observa como la inmensa mayoría de los mayores participantes subrayan la falta de tiempo en el desarrollo de los PI: Es que falta tiempo para lo que queremos hacer. Es falta de tiempo (PMGD21Z). Y, solicitan el aumento de sesiones programadas: Falta que vengan más días. Esa cuestión también es confirmada por los niños: Que vengan otro día para conocer nuevas cosas (N45).

Por otra parte, las personas mayores enfatizan la necesidad de participación por parte de los centros educativos, así como encontrar un punto de unión entre los centros sociales y los colegios: Falta ese enlace. Entre unos y otros. Porque los unos, - venga, a ver si venimos, y los otros, - ya lo veremos... Y luego si no hay tiempo, no se hace (PMGD8J). Afirman que algunos centros no lo consideran relevante: Creen que es mucha complicación (PMGD10J).

Acentuando otros aspectos, se observa que prácticamente la mitad de los mayores participantes consideran importante proyectar nuevas ideas sobre el PI con objeto de mejorarlo y enriquecerlo: Hay que dar un giro, y eso es así, con cosas nuevas que nos vengan bien... Porque al final, lo que queremos es que funcione mejor (PMGD10J); mejorar ciertos aspectos tras la finalización del programa: A veces hay cosas que sabes que no funcionan, por eso se tienen que hablar cuando acaba el programa para cambiarlo (PMGD12J). Del mismo modo, en el ámbito de los profesionales se manifiesta la importancia de fortalecer y mejorar el trabajo interdisciplinar, así como la coordinación institucional: La falta de comunicación inter-centros (EPF7). Los mayores también lo corroboran: Es decir, este centro con otros de Murcia, con uno de Cartagena y con uno de cada parte (PMGD9J). Además, consideran necesario ofrecer el PI fuera del horario lectivo, y a ser posible, enfocado hacia los adolescentes, porque se encuentran en una edad crítica: Más gratificante quizás la labor en los institutos. Lo veo quizás que ahi se pueda conseguir algo más. Es una edad muy crítica (PMGD1A). 
Dando respuesta a las necesidades administrativas, se constata cómo la totalidad de mayores y profesionales reclaman más medios de promoción e implicación desde la administración: $Y$ dé los medios suficientes para eso (...) y lo promocione (PMGD4A). También desde el ámbito político administrativo, por restar valor al hecho intergeneracional: Estos políticos no saben lo importante que es la relación de los críos con nosotros (PMGD21Z). Por otra parte, cerca de la mitad de los mayores participantes, enfatizan la necesidad de una disciplina que incorpore las relaciones intergeneracionales: De decir, esto es así y tiene que formar parte de una disciplina (PMGD3A). Asimismo, reclaman una figura coordinadora: Falta como una figura que represente los PI y que coordine a nivel regional los programa para que pueda llegar a todos los centros (PMGD3A), de la cual, los profesionales recalcan, por unanimidad, incorporar personal técnico especializado: La falta de un profesional que se dedique en exclusividad a este tipo de actividades (EPF17).

En cuanto a las necesidades económicas, los mayores exponen la dificultad de presupuesto y la falta de subvención para desarrollar adecuadamente los PI en su conjunto: Muchas veces las ayudas económicas van donde tienen que ir, pero de lo que hacemos aquí, parece que nadie se acuerda (PMGD21Z). Lo mismo declaran los profesionales: La mejora, en todo caso siempre va unida a la disponibilidad presupuestaria (EPF9).

\section{DISCUSIÓN Y CONCLUSIONES}

El presente estudio centrado en la perspectiva de sus protagonistas, incorpora dos nuevos focos de análisis dirigido a personas mayores y niños como participantes de Pl; ofreciendo una continuidad respecto a otras investigaciones anteriores (Gutiérrez 2010; Gutiérrez y Hernández, 2013). Referente a los motivos de participación de las personas mayores, se evidencia el hecho de sentirse útiles, seguir aprendiendo y satisfacción personal, coincidiendo con el enfoque pedagógico estudiado por Aparicio (2013) y el carácter de necesidad para seguir aprendiendo, expresado por Morón (2014). En esta perspectiva, cobra sentido el modelo sociocrítico para romper con estilos tradicionales que encasillan a los mayores como inactivos e improductivos, como evidenciaron otras investigaciones (García Mínguez, 2002; Martínez de Miguel y Escarbajal de Haro, 2009). En cuanto al rol desempeñado por los mayores como transmisores de conocimiento, los 
datos obtenidos convergen con los estudios de Yuni y Urbano (2005), Gutiérrez, (2011) y Caride (2013), donde los mayores consideran su papel con una actitud protagonista y activa.

Aunque los mayores participan en la propuesta y elaboración de actividades, la dirección de los PI compete a los profesionales coordinadores; esta situación difiere respecto al estudio comparativo llevado a cabo por Kaplan y Larkin (2004), donde los mayores desarrollan el PI con niños, y los coordinadores actúan como apoyo.

El estudio realiza nuevas aportaciones para los mayores, en cuanto al valor de poder renovar sus aprendizajes para adaptarse mejor a la sociedad. Dichos resultados revelan una clara coherencia con las investigaciones de Hatton-Yeo, Klerq, Ohsako y Newman (2001) y Pinazo (2009). La percepción resultante va en la misma dirección que mantienen De-Juanas, Limón y Navarro (2013) y Salmerón, Martínez de Miguel y Escarbajal de Haro (2014), al expresar nuevas formas de desarrollo en los mayores para seguir estando activos, continuar formándose, enfrentarse al aislamiento y mejorar su calidad de vida.

En el caso de los niños entrevistados que interactúan con mayores, aparecen beneficios relacionados con el conocimiento y la experiencia que les aportan; y su percepción hacia los mayores es positiva una vez realizado el PI. (Al respecto, hay que recordar la percepción negativa y estereotipada previa a los PI). Algunas investigaciones también lo han demostrado (Abrams, Eller \& Bryant, 2006; Aday, Sims, McDuffie \& Evans, 1996; Foster, 1997; Rebok et al., 2004; Rosebrook, 2006); todas argumentan que el contacto con las personas mayores es capaz de reducir estereotipos negativos proyectados desde los niños y los jóvenes.

El último bloque de resultados llevado a discusión, hace referencia a las principales necesidades encontradas en el proceso que engloba el funcionamiento de PI. Con referencia a los profesionales, una de las grandes preocupaciones recabada de los resultados se refiere al inexcusable trabajo interdisciplinar, así como la coordinación institucional y la necesidad de implantar una evaluación sólida y una red de centros para trabajar tanto a nivel regional como nacional. Esta radiografía demuestra uno de los retos más significativos acentuado por Kuehne (2003) y la urgencia del trabajo inter-centros (Kaplan, Higdon, Crago \& Robbins, 2004). Con respecto a la evaluación, los resultados coinciden con Gutiérrez (2010), ó con el análisis reflexivo de Domínguez (2012), en cuanto a los componentes fundamentales de un PI. Sobre el ámbito educativo se manifiesta claramente la necesidad de incluir los PI en las programaciones ordinarias de los 
centros de Educación Primaria y Secundaria, como igualmente dedujeron de sus estudios Friedman (1999) y Gutiérrez y Alcaraz (2012). Otro aspecto reclamado por mayores y coordinadores se refiere al aumento de recursos humanos y la deficitaria dotación económica, resultados también observados en estudios como el de Sánchez, Díaz, Doblas, Pinazo y Sáez (2008), y Domínguez (2012).

Una vez expuesta la discusión, y teniendo en cuenta los objetivos planteados en la investigación, se exponen a continuación las principales conclusiones del estudio.

Atendiendo al primer objetivo específico, reconocer y analizar los programas existentes, se constata una enorme variedad de grupos de edad a los que se dirigen los PI reconocidos en la Región de Murcia; abarcando niños de Educación Infantil y Primaria, jóvenes de Educación Secundaria y Universitaria; en alguna ocasión, grupos con discapacidad. Dicho lo cual, aunque se efectúan interacciones con todos los cohortes de participación mencionados, la idea que se desprende de los protagonistas apunta a los niños de Educación Primaria como el grupo de edad con el que tienen más afinidad, contacto y experiencia de trabajo. Concretamente, el Centro escolar es el contexto habitual para el desarrollo de la mayoría de las actividades programadas. Ajustándose a este foco de estudio, se descubren de manera muy significativa programas y actividades basadas en la aportación que realizan los mayores: su experiencia, formas de vida en la infancia (juegos tradicionales, fabricación de juguetes antiguos...), la juventud, los oficios, como eran sus padres y ancestros, etc. El eje vertebral de este programa se denomina "Taller de la experiencia", que viene reconocido en el área de actuación sociocomunitaria dentro de los Centros Sociales.

Otro aspecto relevante se refiere a que el conjunto de actividades propuestas por los profesionales coordinadores tienen entidad propia como "programa" a pesar de que pueda reducirse a actividades puntuales por razones estructurales. Asimismo, el análisis de resultados permite ver que la iniciativa en cuanto a organización y promoción de los PI proceden mayoritariamente de las instituciones de mayores, sin olvidar la coordinación con los responsables del resto de instituciones implicadas para matizar las propuestas de actuación. Y, en cuanto a los motivos de participación en los programas, resaltar que, en el caso de los mayores, se debe principalmente a su satisfacción personal y al cariño que sienten hacia los niños; ven una oportunidad de conocer su mundo y poder transmitirles su conocimiento; descubren una manera de sentirse útiles, poder renovarse y compartir su experiencia con el propio colectivo de personas mayores y el resto de 
generaciones. Esta conclusión se refuerza con las opiniones de los profesionales hacia los mayores, en el sentido de que vivencian un sentimiento de utilidad; motivación y crecimiento de autoestima.

Respecto al segundo objetivo, referido a roles desempeñados, la principal función expresada por los mayores es transmitir su conocimiento y experiencia a los niños con los que comparten el programa. Asimismo, forman parte de la organización y planificación de los programas llevados a cabo, eligiendo, preparando y matizando sus propias actividades para su posterior exposición. No obstante, los temas referidos al modelo de actividad, son propuestos, planificados y supervisados generalmente por los propios coordinadores. A este parecer, las respuestas de los profesionales entrevistados también garantizan, salvo algunas excepciones, una implicación muy considerable de los mayores durante todo el proceso de las acciones intergeneracionales, teniendo siempre presente la guía del técnico responsable.

En la misma tesitura se descubren varios puntos de vista de la imagen percibida por parte de los niños hacia los mayores, pero teniendo en cuenta distintos momentos de interacción en los Pl: algunos estereotipos y visiones negativas previas y gran mayoría de visiones positivas tras la realización de los programas. Se destaca, por tanto, que la percepción de los niños y jóvenes puede cambiar favorablemente una vez interaccionado y convivido con los mayores. Otro aspecto vinculante, en cuanto a la percepción de los profesionales hacia los niños, en su interacción con lo mayores, se constata que este tipo de programas están siendo valiosos para romper con estereotipos persistentes en el contexto social y suplantar ciertos tópicos, fortalecer valores familiares y sociales, enriquecerse mutuamente de sus similitudes y diferencias, así como provocar un acercamiento cultural y de encuentro en espacios comunes.

En cuanto al tercer objetivo, tendente a identificar posibles carencias en los Pl, entre los profesionales aparece con fuerza la necesidad de formación específica; sostienen que, a pesar de adquirir una formación progresiva tras sus años de experiencia, muchos de ellos siguen teniendo la necesidad de cualificación en este ámbito, sobre todo, en técnicas relacionadas con la evaluación. Del mismo modo, creen conveniente que los mayores también reciban una formación previa en el ámbito de desarrollo de programas. 
Otra preocupación por parte de los profesionales viene determinada por la regeneración del trabajo interdisciplinar y la coordinación institucional, aludiendo, a un necesario proceso de evaluación técnica, así como la falta de comunicación entre los diferentes centros organizadores de PI. Aquí los mayores refuerzan la idea de los profesionales con la demanda de un canal comunicativo o idea de coordinación a través de una red inter-centros; ello permitiría compartir nuevas experiencias y contrastar con otras instituciones la forma de actuación, sus ideas, opiniones, etc., para mejorar la calidad de los mismos. Otra necesidad es la falta de sensibilización por parte de la administración pública para el apoyo a la promoción de Pl; consideran indispensable un mayor impulso e implicación desde los órganos políticos para ampliar la cobertura en esta línea de trabajo. Se declara que no hay conciencia real o se olvida reflexionar acerca del nivel funcional y valorativo de la intergeneracionalidad, así como los beneficios que pueden proporcionar la práctica de este tipo de actividades en el horizonte personal y sociocomunitario.

Otros déficits asociados tienen que ver con el apoyo presupuestario y la dotación económica, que frecuentemente es bastante limitada en comparación con actividades emprendidas en otros campos de actuación. Por otra parte, se considera fundamental aumentar la incorporación de personal técnico especializado para ofrecer mayor riqueza, reforzar y desplegar esta línea de trabajo, así como la creación de una figura coordinadora y una disciplina que recoja las relaciones intergeneracionales. Se debe subrayar también por parte de los mayores, la falta de tiempo para poder desarrollar las sesiones del programa, desembocando en una restricción del número de actividades. Por ello, se reclaman más sesiones de encuentro por actividad programada. Aquí se descubre que uno de los motivos de reducción temporal viene determinado por el horario escolar del alumnado y la sobrecarga del profesorado. Generalmente no se oponen, pero en ocasiones lo van dejando en un segundo plano, no prestando a los PI la importancia que merecen. Los mayores insisten en que, si se aumenta el tiempo de dedicación a los programas, se podría dar cobertura a más colegios. Del mismo modo, los niños también exteriorizan el deseo de prolongar más días las actividades porque las consideran cortas y son realmente gratificantes. Con todo lo anterior, se detecta la necesidad de implicación por parte de la comunidad educativa para encontrar un punto de unión y equilibrio entre las diferentes entidades participantes. Si bien se confirma que el mayor peso de los programas está sustentado por los coordinadores y mayores pertenecientes a los Centros sociales, se debe dar argumentos y medios a los colegios para que se impliquen de manera activa. A este 
parecer, se constata el deseo de que la comunidad escolar tome más conciencia en este tipo de actividad, priorizándola con la misma fuerza que otro modelo de programas ya afincados en la programación anual del centro.

\section{REFERENCIAS}

Abrams, D., Eller, A. \& Bryant, J. (2006). An age apart: The effects of intergenerational contact and stereotype threat on performance and intergroup bias. Psychology and Aging, 21 (4), 691-702.

Aday, R. H., Sims, C., McDuffie, W., \& Evans, E. (1996). Changing children's attitudes toward the elderly: the longitudinal effects of an intergenerational partners program. Journal of Research in Childhood Education, 10, 143-151.

Aparicio, C. (2013). Educación y envejecimiento activo. Una experiencia comunitaria. [Tesis Doctoral]. Departamento de Ciencias de la Educación. Universidad de Alcalá.

Baschiera, B., Deluigi, R. \& Luppi, E. (2014). Educazione intergenerazionale. Prospettive, progetti e metodologie didáctico-formative per promovere la solidaritá fra le generazioni. Milano: F. Angeli.

Caride, J. A. (2013, Octubre). La Educación Social en el Siglo XXI como instrumento para la construcción de una nueva ciudadanía. Conferencia inaugural presentada a las Jornadas "Influencia de la educación y la sensibilización social en la construcción de una sociedad para todas las edades", Madrid, 1 y 2 de octubre de 2013.

De-Juanas, A., Limón, M. R., y Navarro, E. (2013). Análisis del bienestar psicológico, estado de salud percibido y calidad de vida en personas adultas mayores. Pedagogía Social. Revista Interuniversitaria, 22, 153-168.

Del Valle, G. y Coll, L. (2011). Relaciones Sociales y Envejecimiento Saludable. Institut de l'Envelliment de la Universitat Autònoma de Barcelona FICE-UAB: Fundació Agrupació Mútua.

Domínguez, M. E. (2012). Programas intergeneracionales: Reflexiones generales a través del análisis Dafo. Revista Electrónica de Psicología Social «Poiésis», 24, 2-15.

Foster, K. (2007). Creating a child care center in a nursing home and implementing an intergenerational program. United States: Eric Document Reproduction Service: $\mathrm{ED}$.

Friedman, B. M. (1999). Connecting generations: integrating aging education and intergenerational programs with elementary and middle grades curricula. Needham Heights, MA: Allyn y Bacon.

García Mínguez, J. (2002). Introducción: una aproximación al concepto de educación intergeneracional. En J. García y M. Bedmar (Eds.), Hacia la educación intergeneracional (pp. 11-22). Madrid: Dykinson. 
Granville, G. y Hatton-Yeo (2002). Intergenerational engagement in the UK: A framework for creating inclusive communities. En M. Kaplan, N. Henkin, N. y A. Kusano (eds). Linking lifetimes: a global view of intergenerational Exchange. Lanham, MD: University Press of América, pp. 193-208.

Gutiérrez, M. (2010). Los programas intergeneracionales en la Región de Murcia. Análisis de la situación y propuestas de mejora [Tesis Doctoral]. Departamento de Teoría e Historia de la Educación. Facultad de Educación. Universidad de Murcia.

Gutiérrez, M. (2011). Programas intergeneracionales. Teoría, política y práctica. Saarbrücken, Alemania: Editorial Académica Española.

Gutiérrez, M. y Alcaraz, S. (2012). Las relaciones intergeneracionales en el aula. Una oportunidad para la atención a la diversidad en Educación Primaria. En G. PérezSerrano (Ed.), I Simposio Internacional de Envejecimiento Activo y Solidaridad Intergeneracional. [CD-ROM] Madrid: UNED.

Gutiérrez, M. y Hernández, D. (2013). Los beneficios de los programas intergeneracionales desde la perspectiva de los profesionales. Pedagogía Social. Revista Interuniversitaria $21,213-235$.

Hatton-Yeo, A. (2009). Algunos principios generales del desarrollo comunitario intergeneracional. Espai Social, Revista del colegio oficial d'educadores i educadors socials de la comunitat valenciana, 9, 21-24.

Hatton-Yeo, A. Klerq, J., Ohsako, T., y Newman, S (2001). Política pública y recomendaciones para la investigación: una perspectiva internacional. En A. Hatton-Yeo y T. Ohsako (Eds.), Programas Intergeneracionales: política pública e implicaciones de la investigación. Una perspectiva internacional (pp. 9-17). Hamburgo: Instituto de la UNESCO para la Educación.

Hatton-Yeo, A. y Ohsako, T. (2001). Programas Intergeneracionales: política pública e implicaciones de la investigación. Una perspectiva internacional. Hamburgo: Instituto de la UNESCO para la Educación.

Internacional Consortium of Intergenerational Programmes (ICIP) (1999). Consorcio Internacional para los Programas Intergeneracionales $(s, f)$. Recuperado de http://www.icip.info/

Kaplan, M., Higdon, F., Crago, N., \& Robbins, L. (2004). Futures festival: an intergenerational strategy for promoting community participation. Journal of Intergenerational Relationships, 2 (3/4), 119-146.

Kaplan, M. y Larkin, L. (2004). Launching Intergenerational Programs in Early Childhood Settings: A Comparison of Explicit Intervention with an Emergent Approach. Early Childhood Educational Journal, 31 (3), 157-163.

Kuehne, V. S. (2003). The state of our art: Intergenerational program research and evaluation: Part one. Journal of Intergenerational Relationships, 1 (1), 145-161.

MacCallum, J., Palmer, D., Wright, P., Cumming-Potvin, W., Northcote, J., Booker, M. \& Tero, C. (2006). Community building through intergenerational exchange programs. Australia: National Youth affairs Research Scheme. 
Martínez de Miguel, S. y Escarbajal de Haro, A. (2009). Alternativas socioeducativas para las personas mayores. Madrid: Dykinson.

Martínez de Miguel, S., Moreno, P. y Escarbajal, A. (2017). Envejecimiento activo, programas intergeneracionales y educación social. Madrid: Dykinson.

McCrea, J. \& Smith, T. (1997). Social Issues Addressed by Intergenerational Programs. En S. Newman; C. Ward; T. Smith, J. Wilson y J. McCrea (eds.). Intergenerational Programs. Past, Present and Future (pp.37-51).Washington: Taylor \& Francis.

Morón, J. A. (2014). Educación y personas mayores. Revista Electrónica Interuniversitaria de Formación del Profesorado, 17 (1), 107-121.

Newman, S. y Sánchez, M. (2007). Los programas intergeneracionales: concepto, historia y modelos. En M. Sánchez (Ed.), Programas intergeneracionales: una sociedad para todas las edades (pp. 37-69). Barcelona: Colección Estudios Sociales Obra Social "la Caixa".

Organización Mundial de la Salud (2002). Active Aging. Geneva: WHO. Recuperado de http://www.who.int/es/

Pinazo. S. (2009). Beneficios de los programas intergeneracionales. Espai Social, Revista del Colegio Oficial d'Educadores i Educadors Socials de la Comunitat Valenciana, 9, 13-16.

Pinazo, S. (2012). Las personas mayores proveedoras de conocimientos y cuidados. El papel de los programas intergeneracionales. Educación Social: Revista de Intervención Socioeducativa, 51, 45-66.

Rebok, G. W., Carlson, M. C., Glass, A., McGill, S., Hill, J., Wasik, B., lalongo, N., Frick, K. D., Fried, L. P., \& Rasmussen, M. D. (2004). Short-Term Impact of Experience Corps Participation on Children and Schools: Results a Pilot Randomized Trial. Journal of Urban Health, 81 (1), 79-93.

Rosebrook, V. (2006). Intergenerational interactions Enhance Young Children's PersonalSocial Skills. The Generations United Magazine, 11 (2), 5-7.

Salmerón, J. A., Martínez de Miguel, S., y Escarbajal, A. (2014). Vejez, mujer y educación. Un enfoque cualitativo de trabajo socioeducativo. Madrid: Dykinson.

Sánchez, M., Díaz, P., López, J., Pinazo, S. y Sáez, J. (2008). INTERGEN. Descripción, análisis y evaluación de los programas intergeneracionales en España. Modelos y buenas prácticas. Resumen ejecutivo. Recuperado el 6-9-2012 de http://www.imserso. es/InterPresent1/groups/imserso/documents/binario/idi172 06ugranada.pdf

Serdio, C., Díaz, B., y Cifuentes, P. (2013). Envejecer activamente, aprender activamente. Apuntes para una propuesta educativa en el ámbito universitario. Revista de Psicología, 2 (1), 91-98.

Villas-Boas, S., Oliveira, A. L., Ramos, N. \& Montero, I. (2015). Conhecimento da comunidade local para a elaboração e implementação de programas intergeracionais. Rev. Bras. Est. Pop., Rio de Janeiro, 32 (1), 189-197.

Yuni, J. A. y Urbano C. A. (2005). Educación de adultos mayores: teoría, investigación e intervenciones. Córdoba (Argentina): Brujas. 
